\title{
Improving the Efficiency of Pattern Extraction for Character Projection Lithography using OPC Optimization
}

\author{
Hirokazu Nosato, Hidenori Sakanashi, \\ Masahiro Murakawa and Tetsuya Higuchi \\ National Institute of Advanced Industrial Science and Technology (AIST)
}

Japan

\section{Introduction}

In recent years, with the growth in demand for digital information appliances, system LSI devices for digital information appliances are becoming the mainstream in the semiconductor industry, replacing LSI devices for PCs. For such system LSIs, it is important to be able to manufacture system LSI devices with multi-functionality and high performance with high-variety, low-volume production processes incurring low costs and short turnaround-times (TAT). However, as the sizes of semiconductor devices become increasingly smaller, the accompanying increases in mask costs and TAT are major obstacles preventing rapid advances in the development of digital information appliances with system LSIs. These problems are due to the increasing complexity and data volume of mask patterns. Similarly, while Optical Proximity Correction (OPC) can correct a mask pattern to improve the fidelity of the projected image pattern on the wafer, the method involves huge calculation loads (including runtime, data volume, and processing costs) as the calculations required to correct a mask pattern become ever more complex.

In order to solve mask costs and TAT problems, Character Projection Lithography (CPL) has been devised as a high-throughput electron-beam direct-write technology (Nakayama et al., 1990). CPL has the advantage of reducing the number of electron beam (EB) shots compared to conventional variably-shaped beam lithography (Pfeiffer, 1978), because each character pattern that appears frequently in a layout can be simultaneously written by a single EB shot with a CP aperture mask. In order to achieve such reductions in the number of EB shots, it is important to extract frequently-used character patterns and to prepare $\mathrm{CP}$ aperture masks. However, when CPL is applied to OPCed layouts for a system LSI, while CPL can work in reducing the number of EB shots in the memory device, CPL cannot function with the random logic device. Because random logic devices are composed by laying out logic cells randomly, one character pattern can be deformed into many different patterns to correct the Optical Proximity Effect (OPE) from neighboring patterns, and thus require complicated $\mathrm{OPC}$, so they cannot be extracted as a unique CP aperture mask.

In order to overcome the problems of realizing CPL with a random logic device, we propose a method of improving the efficiency of pattern extraction by employing an adaptive OPC method (Matsunawa et al., 2005; Matsunawa et al., 2007). As a new OPC calculation model Source: Lithography, Book edited by: Michael Wang,

ISBN 978-953-307-064-3, pp. 656, February 2010, INTECH, Croatia, downloaded from SCIYO.COM 
designed to achieve high-precision correction and reductions in OPC runtimes by reducing the calculation domain, the adaptive OPC method has two important characteristics. One is that OPC is applied to each cell before layout design. Thus, the adaptive OPC method can be regarded as being a cell-based OPC method. The other characteristic is the optimization of variable OPC features in peripheral regions around the OPCed cell due to the OPE from neighboring patterns after layout design.

In this study, we enhance the basic adaptive OPC method by developing two important algorithms: (1) a cell grouping algorithm that categorizes deformed cells and extracts some typical cell groups, and (2) an OPC optimization algorithm that regards the cells in a group as one typical cell and corrects the OPC features of the typical cell to form a CP aperture mask. In conducted verification experiments with two full-chip level OPCed layouts based on actual functional LSI, we successfully achieve an improvement of approximately $60 \%$ in extraction efficiency.

This chapter is organized as follows: In Section 2, the problems of realizing CPL with random logic devices are briefly described. Section 3 introduces our proposed improvement method. Section 4 presents the details and results of conducted experiments for a feasibility study and Section 5 shows the results of verification experiments with actual functional LSI data. Finally, Section 6 discusses the results and draws some conclusions.

\section{The problems of realizing CPL with random logic devices}

$\mathrm{CPL}$ is a promising technology as a high-throughput electron-beam direct-write technology. The advantage of CPL is that it has a lower number of electron beam (EB) shots compared to conventional variably-shaped beam lithography, because character patterns that appear frequently in a layout can be simultaneously written by a single EB shot with a CP aperture mask. Accordingly, it is important to extract the sets of frequently-used character patterns and to prepare $\mathrm{CP}$ aperture masks in order to reduce the number of EB shots.

However, it has been reported by ASET (ASET, 2006) that although CPL can be beneficial for the memory device in reducing the number of EB shots, CPL cannot function on random logic devices. According to the report for a poly-silicon layer, the single character pattern referred to 1539 times in the memory device is diversified to eight deformed patterns with OPC features. On the other hand, the character pattern referred to 680 times in the logic device is diversified to 660 deformed patterns with OPC features. The reason for the difference in the number of the deformed patterns is that random logic devices are composed by randomly laying out logic cells, and one character pattern can be deformed into many different patterns due to the Optical Proximity Effect (OPE) from neighboring patterns. That is a character pattern in random logic devices will be deformed into many different patterns that require complicated OPC features, and so they cannot be extracted as a unique $\mathrm{CP}$ aperture mask. Thus, in order to realize the advantage of CPL to reduce the number of EB shots with random logic devices, the novel OPC method is required to improve the efficiency of pattern extraction with moderate levels of diversification.

\section{Improving pattern extraction efficiency for CPL}

\subsection{Approach}

In order to overcome the problems of realizing CPL with a random logic device, we propose a method of improving the efficiency of pattern extraction by employing an adaptive OPC 
method (Matsunawa et al., 2005; Matsunawa et al., 2007). As a new OPC calculation model designed to achieve high-precision correction and reductions in OPC runtimes by reducing the calculation domain, the adaptive OPC method has two important characteristics. One is that OPC is applied to each cell before layout design. Thus, the adaptive OPC method can be regarded as being a cell-based OPC method. The other characteristic is the optimization of variable OPC features in peripheral regions around the OPCed cell due to the OPE from neighboring patterns after layout design.

In this research, we enhance the basic adaptive OPC method by developing two important algorithms: (1) a cell grouping algorithm that categorizes deformed cells and extracts some typical cell groups, and (2) an OPC optimization algorithm that regards the cells in a group as one typical cell and corrects the OPC features of the typical cell to form a CP aperture mask. Fig. 1 illustrates the basic concept of the proposed method with these two algorithms. $A$ character cells are extracted from a cell layout data. Suppose that the $A$ character cells would be deformed into five individual cells $A_{1} \sim A_{5}$ after OPC processing. With the cell grouping algorithm, the deformed cells are extracted as two typical cell groups $A_{1} \sim A_{3}$ and $A_{4} \sim A_{5}$. The OPC optimization algorithm optimizes a typical cell, $A a$ or $A b$, for each cell group based on the cells within each group.

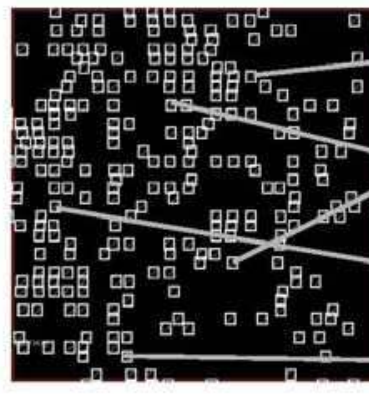

Extracted character cells on the layout data (70بm square)

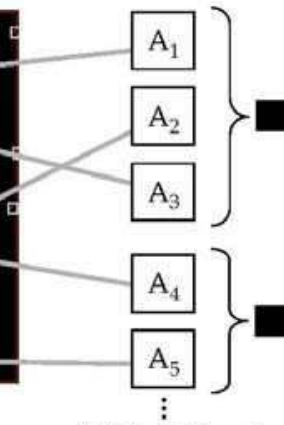

With different environments of neighboring patterns

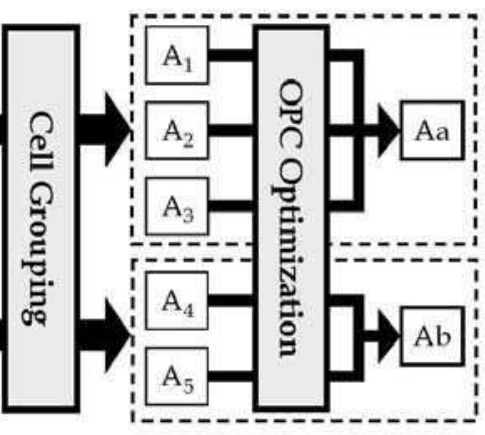

Optimized typical cells

Fig. 1. Framework of the proposed method.

\subsection{The cell grouping algorithm}

This subsection describes the cell grouping algorithm which can extract a typical cell group. The character cells are inserted into all of the different neighboring patterns. A neighboring pattern refers to a specific layout situation relating to crude density, directionality and design in the peripheral regions of a character cell, as shown in Fig. 2. The OPC features of a character cell are affected by the OPE from the neighboring region. For example, if two character cells are placed in similar neighboring patterns, there will be similarities in their OPC features which can be optimized by the OPC optimization method to produce a typical cell. Thus, it is possible to identify typical cell groups that have similar OPC features by analyzing the neighboring patterns.

In this research, we propose a cell grouping algorithm that involves a two-stage processing of categorizing cells in terms of layout direction and of comparing overlapping patterns by 
analyzing neighboring patterns. Fig. 3 presents the flowchart for the proposed cell grouping algorithm. In preparation, data for the character cells and their neighboring patterns are loaded from the layout data. In the first stage, the character cells are roughly categorized into several provisional cell groups according to the directionality of neighboring patterns. In the second stage, the multiple cell groups are finely categorized into typical cell groups that can be optimized as one typical cell by comparing the overlaps for neighboring patterns. The two categorization methods are explained in detail in the following subsections.

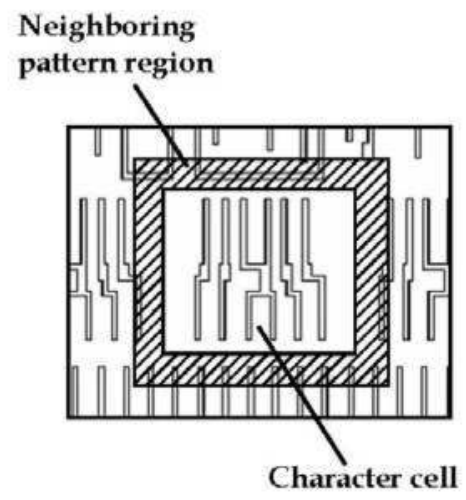

Fig. 2. A character cell with its neighboring pattern. Source: based on Nosato et al., 2007.

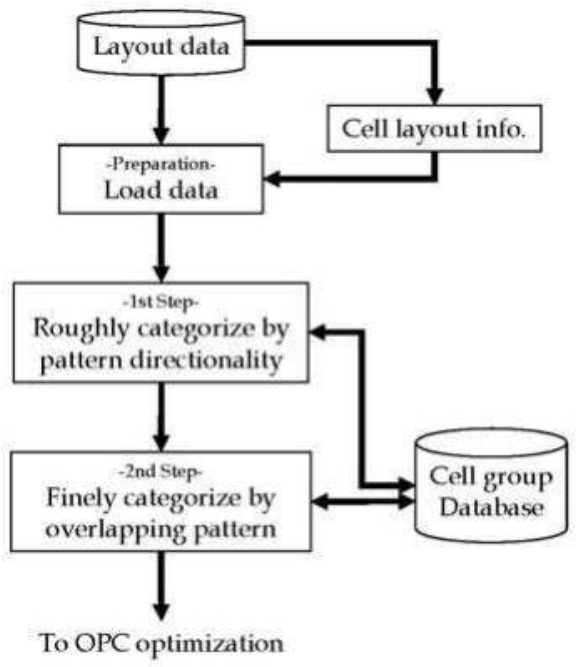

Fig. 3. Flowchart of the cell grouping algorithm.

\subsubsection{The rough categorization method according to directionality}

The rough categorization method generates provisional cell groups that have similar neighboring patterns. The OPE on a character cell depends on the density of neighboring 
patterns. Employing this method, we analyzed the neighboring patterns in our test data (which is described in more detail in Section 4.1), and Fig. 4 and Fig. 5 present parts of the analysis results. Fig. 4 presents the crude density of neighboring patterns in the peripheral regions of a character cell. Neighboring patterns are denser in the vertical region (Fig. 4(a)) than in the horizontal region (Fig. 4(b)). Fig. 5 presents results for the directionality of patterns in the vertical region. The horizontal pattern (Fig. 5(a)) is denser than the vertical pattern (Fig. 5(b)) in the vertical region of the character cell. Therefore, in this research, the character cells are roughly categorized into several provisional cell groups according to the criterion of directionality in the vertical regions, as shown in Table 1.

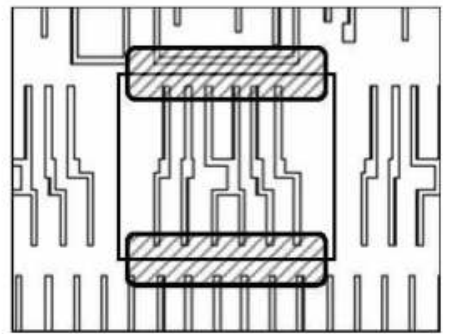

(a) Vertical region of a character cell

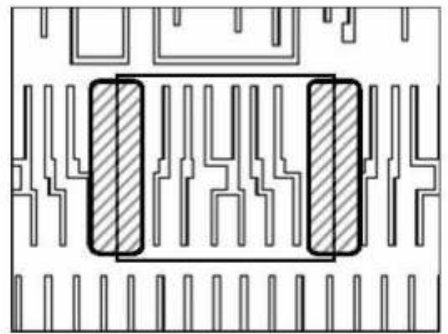

(b) Horizontal region of a character cell

Fig. 4. Crude densities of neighboring patterns. Source: based on Nosato et al., 2007.

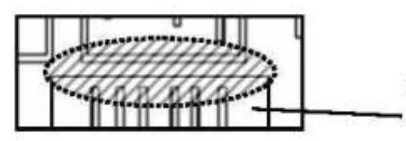

(a) Horizontal patterns

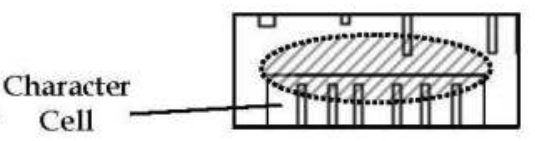

(b) Vertical Patterns

Fig. 5. Pattern directionality in the vertical region. Source: based on Nosato et al., 2007.

\begin{tabular}{|c|c|}
\hline Provisional cell group & Pattern directionality \\
\hline \hline 1 & Horizontal pattern in the upper vertical region \\
\hline 2 & Horizontal pattern in the lower vertical region \\
\hline 3 & No horizontal pattern in the vertical region \\
\hline
\end{tabular}

Table 1. The rough grouping criteria for pattern directionality in the vertical region.

\subsubsection{The fine categorized method according to overlap comparisons}

In order to finely categorize the groups into typical cell groups that can be optimized as a single typical cell, the fine categorization method compares the areas of overlap for neighboring patterns one-by-one for each provisional cell group, as shown in Fig. 6. As Fig. 6 indicates, the overlapping areas show similarities in terms of their neighboring patterns. Therefore, the character cells in the provisional cell groups are finely categorized into typical cell groups based on comparisons for the overlapping areas. 


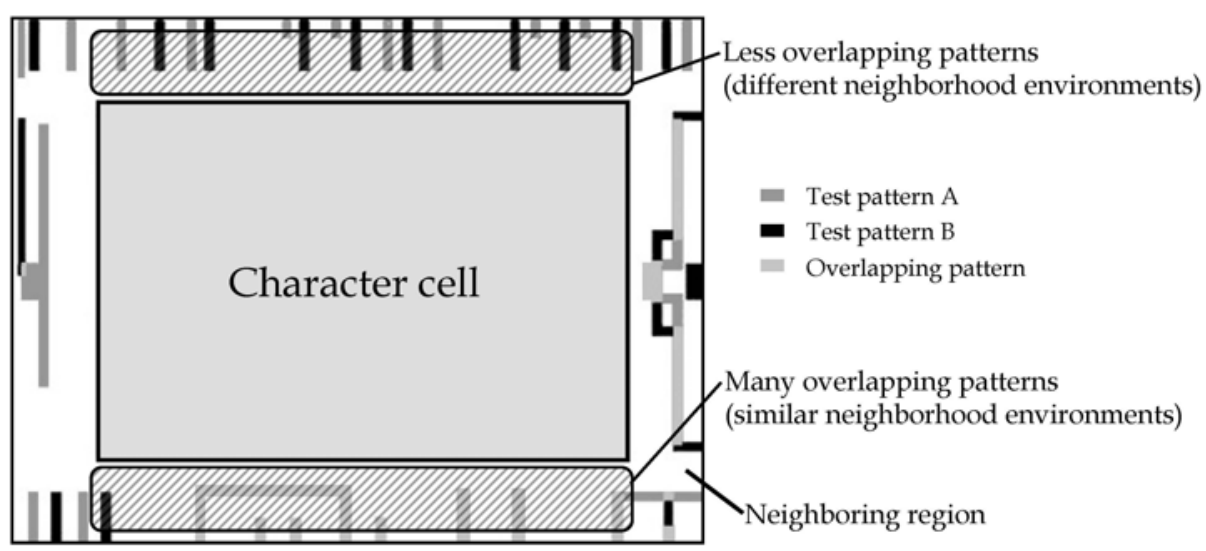

Fig. 6. Comparison of neighboring patterns.

\subsection{OPC optimization algorithm}

This section describes the OPC optimization algorithm which can adjust the OPC features of a categorized cell group to generate one typical character cell. Fig. 7 shows the flowchart for the proposed OPC optimization algorithm. Initially, categorized cell group data is loaded from the database of cell groups. Next, the OPC features of a character cell are coded as common OPC optimization parameters. These parameters are reflected in all character cells within the same categorized cell group. In the first fitness calculation, initial common OPC parameters are evaluated on each neighboring pattern using a lithography simulation.

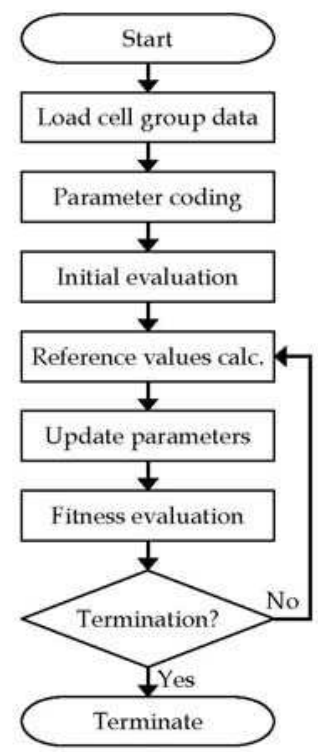

Fig. 7. Flowchart for OPC optimization. 
Then, the algorithm calculates which reference values are better to update to the common parameters, based on the fitness values of each neighboring pattern, and updates the common parameters according to these reference values. Finally, the updated common parameters are evaluated again. When fitness evaluations exceed target accuracy for OPC, or evaluation iterations exceed a predetermined number, then the optimization algorithm terminates. We detail the coding of parameters, the fitness evaluation and the reference values calculations below.

\subsubsection{Coding of optimization parameters}

The common OPC optimization parameters correspond to variables representing the OPC features of an OPCed character cell. The parameters are expressed as a one-dimensional array that consists of the horizontal directions and the vertical directions of the OPC features, as shown in Fig. 8. These parameters represent the polygons for OPC features, and they are expressed as real numbers.

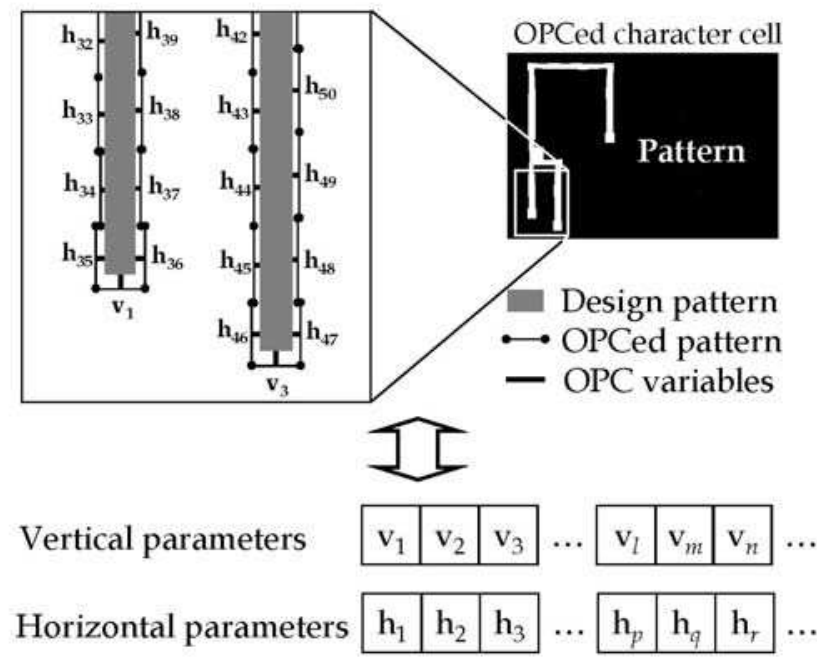

Fig. 8. Coding of optimization parameters.

\subsubsection{Evaluation method}

In this evaluation method, because it is necessary to simultaneously evaluate the character cells in terms of every neighboring pattern within a categorized cell group, we propose parallel processing based on the master-slave model (Cantú-Paz, 1997) for this evaluation, as shown in Fig. 9. Each slave processor is assigned the task of evaluating each neighboring pattern in a lithography simulation. For example, when there are four cells within a categorized cell group, one master processor and four slave processors would be used for evaluation. Fig. 10 presents the flowchart for processing on the master and the slave processors. Fitness is calculated in terms of differences between the target pattern (design layout pattern) and the lithography image output by the lithography simulator as measured at the evaluation points for each OPC feature. 


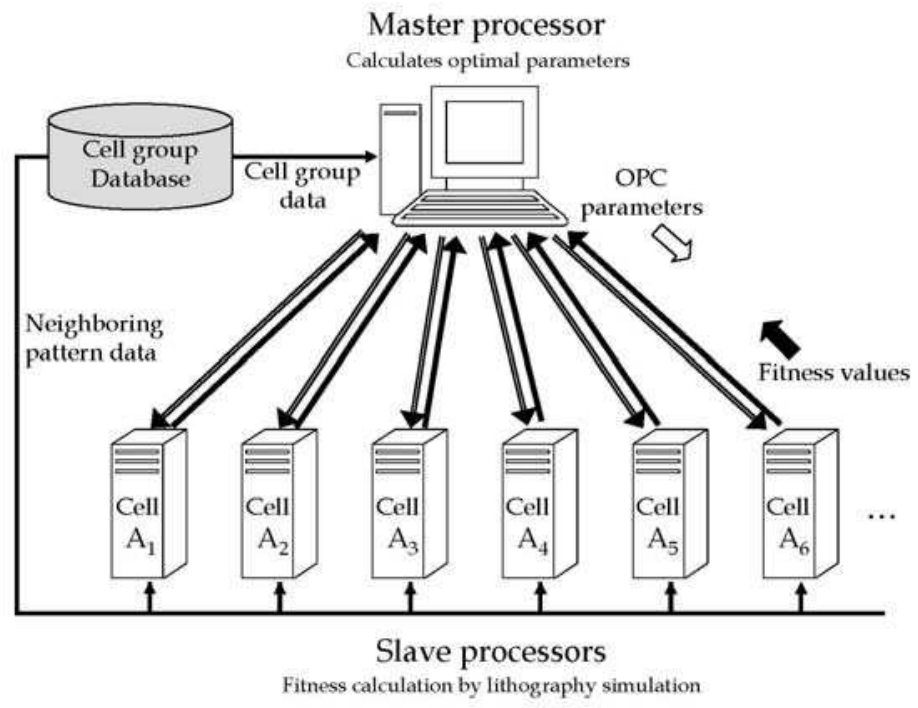

Fig. 9. Parallel processing for OPC optimization.
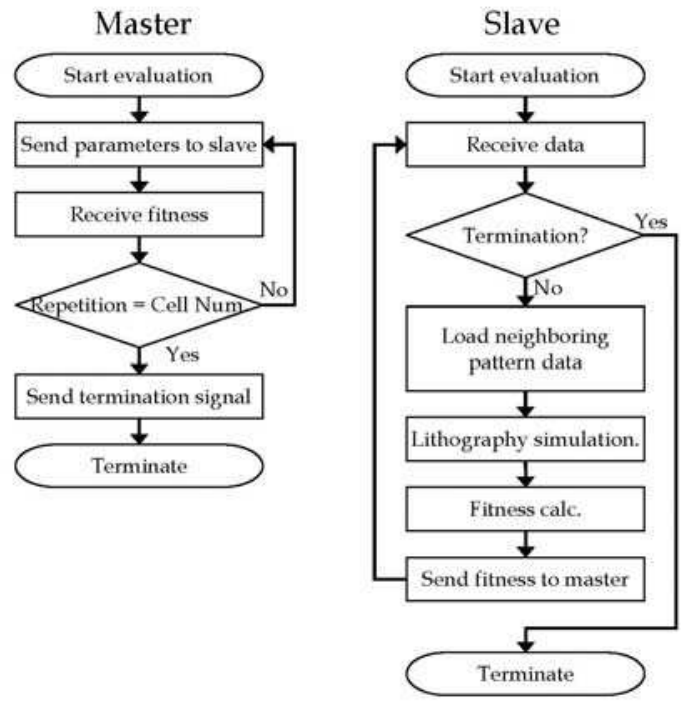

Fig. 10. Flowchart of the evaluation method.

\subsubsection{Calculation of reference values}

The optimization method utilizes one set of common parameters and multiple sets of fitness value of each neighboring parameter. Thus, it can calculate which reference values are better to update to the common parameters, based on the fitness values of each neighboring pattern, and updates the common parameters accordingly to these references values. The calculation generates the reference value $R_{j}$ from the fitness $F_{i j}$ according to the following formula, 


$$
R_{j}=\sigma \cdot \frac{1}{n} \sum_{i=1}^{n} F_{i j}
$$

where $i$ is the index of the cell within a cell group, and $j$ is the position index of the OPC feature for each cell, and $\sigma$ denotes a control factor for optimization accuracy, which is based on the step function shown in Fig. 11. This factor functions to control the adjustment level, such as coarse adjustment with large fitness values or fine adjustment with small fitness values in terms of the OPC features.

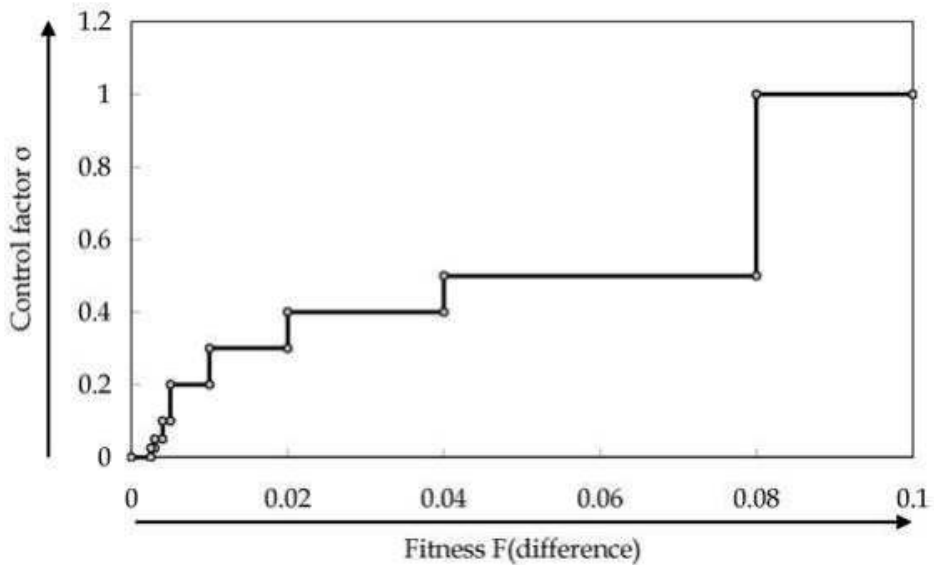

Fig. 11. Control factor for adjustment accuracy.

\section{Experimental results and discussion for the feasibility study}

\subsection{Configuration of the test data}

Evaluation experiments were performed using 40 types of test patterns. The test patterns were based on actual functional cell data from the $130 \mathrm{~nm}$ cell library of STARC (Semiconductor Technology Academic Research Center, Japan). A test pattern consists of one character logic cell in the center and eight logic cells around it, as shown in Fig. 12(a). The character cell was fixed in the center for all 40 test patterns. The eight logic cells were chosen from 26 different logic cells including 13 circuit types, and randomly arrayed around the character cell. That is, arrangements of cell in test patterns imitated different neighboring patterns extracted from the random logic devices, as shown in Fig. 12(b).

During the preparation for the experiments, test patterns according to the 90nm design rule were created by shrinking the $130 \mathrm{~nm}$ cell library arrayed patterns. In addition, the modelbase OPC of Panoramic Technology was applied to the character cell to generate initial OPC parameters as a cell-based OPC before layout.

\subsection{Experimental results for the cell grouping algorithm}

The experimental conditions are presented in Table 2 . The proposed cell grouping algorithm categorized the 40 types of character cells with each test pattern into six typical cell groups. Fig. 13 shows the result tree achieved by the categorization by the cell grouping algorithm. In the first stage of categorization, two provisional typical cell groups were categorized according to the directionality of neighboring patterns, and 19 typical cells were left 


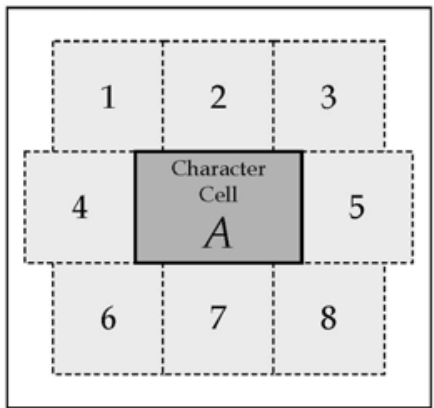

(a) Configuration of test pattern

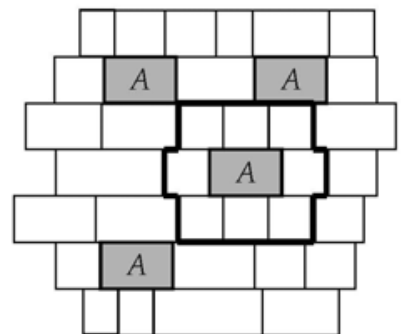

(b) Layout of logic device

Fig. 12. Test pattern for experiments.

\begin{tabular}{|c|c|}
\hline OS & WindowsXP Professional x64 Edition Ver. 2003 Service Pack 2 \\
\hline CPU & Inter ${ }^{\circledR}$ Xeon ${ }^{\mathrm{TM}} 3.00 \mathrm{GHz} \times 2$ \\
\hline Memory & $2 \mathrm{~GB}$ \\
\hline Simulator & Panoramic Technology EM Suite v5.00 \\
\hline Illumination & $\lambda=193 \mathrm{~nm}, k_{1}=0.4, N A=0.85$, Annular $(0.5 / 0.75)$ \\
\hline Accuracy & $0.001 \mu \mathrm{m}$ \\
\hline
\end{tabular}

Table 2. Experimental conditions for cell grouping.

uncategorized. In the second stage of categorization, the six typical cell groups were categorized based on comparisons of the overlapping areas in the neighboring patterns. Finally, based on the 6 typical cell groups, sets of multiple types $(3,2,2,4,5$, and 2 types) of the character cells for each test pattern were extracted, as shown in Table 3. The extracted typical cell groups were separately corrected as one typical character cell in the subsequent OPC optimization experiment.

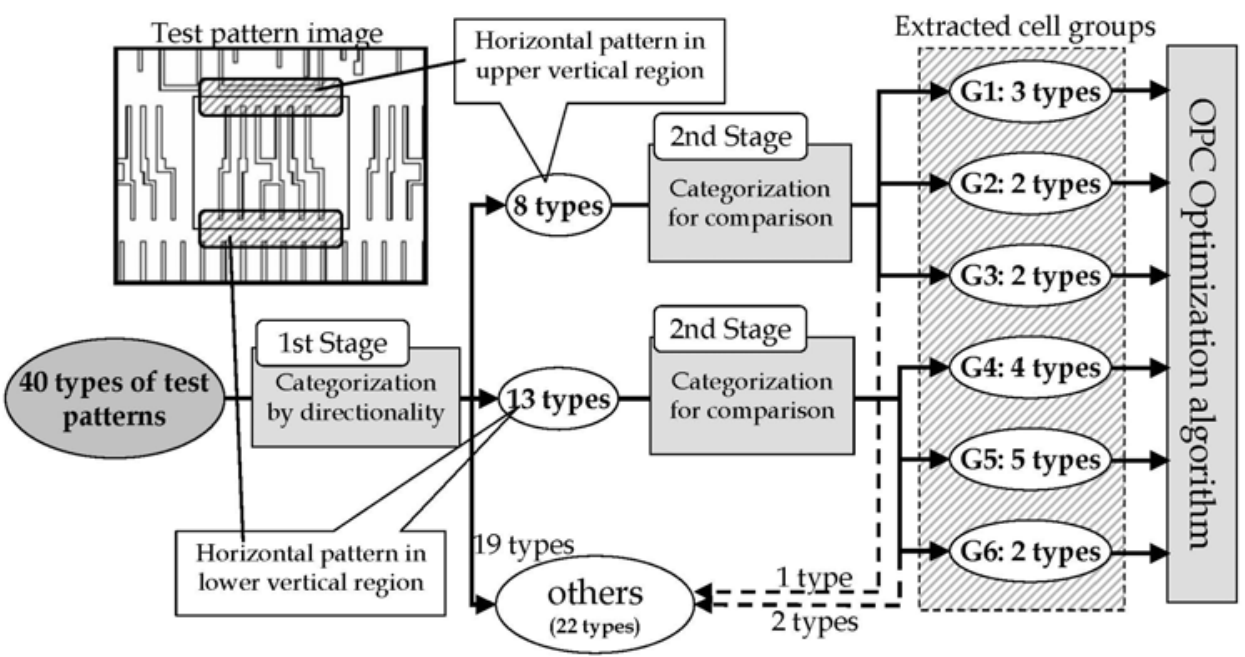

Fig. 13. Categorized tree for cell-group extraction. 


\begin{tabular}{|c|c|c|}
\hline Cell group & Type-count & Number of categorized test patterns \\
\hline G1 & 3 types & $1,6,23$ \\
\hline G2 & 2 types & 3,25 \\
\hline G3 & 2 types & 19,29 \\
\hline G4 & 4 types & $7,13,25,27$ \\
\hline G5 & 5 types & $16,17,26,33,36$ \\
\hline G6 & 2 types & 9,14 \\
\hline
\end{tabular}

Table 3. Categorization results of the experiments.

\subsection{Experimental results for OPC optimization}

The experimental conditions are presented in Table 4. We applied the proposed OPC optimization algorithm to each of the extracted cell groups. The results of the experiments are presented in Table 5 and the graph shows the largest errors between the sizes of target features and the simulated lithography image. Although the largest errors were approximately $34 \mathrm{~nm}$ before optimization, the proposed algorithm decreased the errors to approximately $3 \mathrm{~nm}$. The results before optimization indicate that the application of OPC only for the character cell is insufficient to correct for the character cell within its neighboring patterns. That is, the OPE from neighboring patterns caused differences of approximately $34 \mathrm{~nm}$ between the target pattern and the lithography simulated pattern. The differences were corrected by allowing all members in each typical cell group to have common OPC features. Fig. 14 shows how the OPC features were optimized in the experiment. Clearly, the proposed control factor within the optimization algorithm functioned effectively by realizing coarse adjustment with large fitness values and fine adjustment with small fitness values.

\begin{tabular}{|c|l|}
\hline OS & Redhut Linux v2.6.9.42.ELsmp \\
\hline CPU & Intel® Core $^{\mathrm{TM}} 2$ duo 3.00 GHz $x 2$ \\
\hline Memory & $4 \mathrm{~GB} / 8 \mathrm{~GB}$ \\
\hline Compiler & gcc 3.3 \\
\hline Processor & Up to 6 \\
\hline Communication library & Mpich-1.2.6 build by gcc 3.3 \\
\hline Simulator & Lithography simulator from AIST \\
\hline Illumination & $\lambda=193 \mathrm{~nm}, k_{1}=0.4, N A=0.85$, Annular $(0.5 / 0.75)$ \\
\hline Calculation Area & $5.12 \times 5.12 \mu \mathrm{m}^{2}$ \\
\hline Accuracy & $0.0025 \mu \mathrm{m}$ \\
\hline OPC Parameters & 279 real values \\
\hline Fitness points & 279 points \\
\hline Termination & Repetition of evaluation $=200$ times \\
\hline
\end{tabular}

Table 4. Experimental condition for OPC optimization.

\begin{tabular}{|c|c|c|}
\hline Cell group & Before optimization & After optimization \\
\hline G1 & $34.3 \mathrm{~nm}$ & $2.97 \mathrm{~nm}$ \\
\hline G2 & $34.7 \mathrm{~nm}$ & $2.68 \mathrm{~nm}$ \\
\hline G3 & $34.2 \mathrm{~nm}$ & $2.81 \mathrm{~nm}$ \\
\hline G4 & $34.3 \mathrm{~nm}$ & $2.77 \mathrm{~nm}$ \\
\hline G5 & $34.7 \mathrm{~nm}$ & $2.84 \mathrm{~nm}$ \\
\hline G6 & $34.3 \mathrm{~nm}$ & $3.56 \mathrm{~nm}$ \\
\hline
\end{tabular}

Table 5. Adjustment results for OPC optimization. 


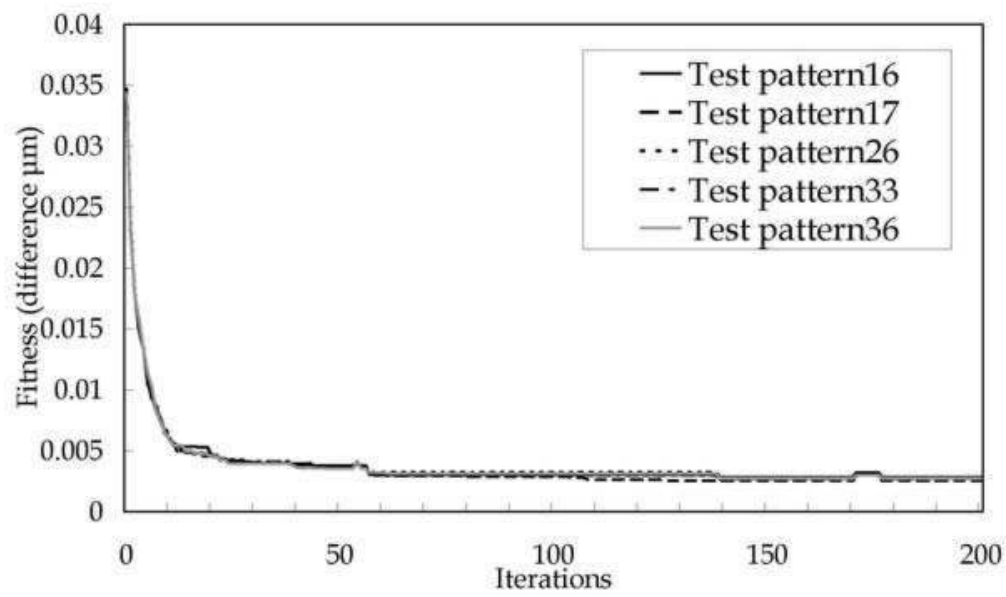

Fig. 14. Fitness as a function of experiment iterations.

\subsection{Discussion}

As the results of the cell grouping experiment and the OPC optimization experiment, the 40 types of character cells with the each test pattern were reduced to six typical character cells together with a set of 22 unique types, making a total of 28 different types of cells. Accordingly, we successfully achieved a $30 \%$ improvement in extraction efficiency. In this research, we proposed a categorization algorithm based on directionality. However, the categorization algorithm remains is a matter for further consideration. If it were possible to discover a more effective approach to categorization, it might be possible to realize further improvements in the pattern extraction efficiency for CPL.

\section{Verification experiments with actual functional LSI data}

\subsection{Configuration of the verification data}

In these verification experiments, we verified the proposed method with two full-chips level OPCed layouts based on the actual functional LSI of STARC (Semiconductor Technology Academic Research Center, Japan). There were two kinds of verification data (Data-1 \& Data-2), which related to clipped 70 square micrometer areas from different OPCed layouts. Because, Data-1 and Data-2 consist of 1,023 cells and 272 cells respectively, they have different features in terms of cell density.

\subsection{Experimental results and discussion}

Fig. 15 presents the experimental results for one cell $A$ for the cell grouping algorithm applied to Data-1. There were 256 cells for cell $A$ within Data-1, as shown in Fig. 15(a). Of these $A$ cells, 198 were categorized into 35 cell groups, which are colored and hatched in Fig. 15(b).

Table 6 presents the experimental results for all cells undergoing the cell grouping algorithm for both Data sets. The details for the entries in Table 6 were as follows:

- Total cells: (1)

Number of all cells in the clipped 70 square micrometer area. 


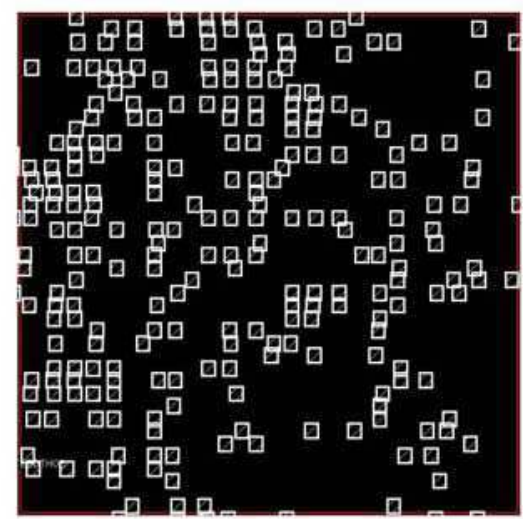

(a) Search result of cell $\mathrm{A}$ in the Data-1

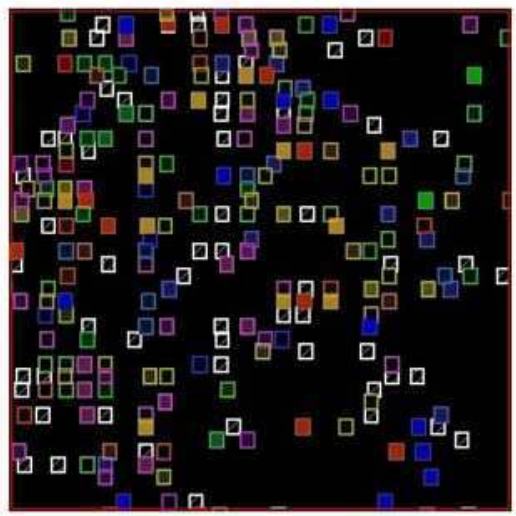

(b) Categorization result of cell A in the Data-1

Fig. 15. Categorization results for cell A in the Data-1.

\begin{tabular}{|c|c|c|c|c|c|c|}
\hline Data & $(1)$ & $(2)$ & $(3)$ & $(4)$ & $(3)+(4)$ & $\{(3)+(4)\} /(1)$ \\
\cline { 2 - 7 } & $\begin{array}{c}\text { Total } \\
\text { cells }\end{array}$ & $\begin{array}{c}\text { Extracted } \\
\text { cells }\end{array}$ & $\begin{array}{c}\text { Unextracted } \\
\text { cells }\end{array}$ & $\begin{array}{c}\text { Cell } \\
\text { groups }\end{array}$ & $\begin{array}{c}\text { Cell } \\
\text { types }\end{array}$ & $\begin{array}{c}\text { Rate of } \\
\text { reduction }\end{array}$ \\
\hline \hline Data-1 & 1023 & 826 & 197 & 206 & 403 & $39.4 \%$ \\
\hline Data-2 & 272 & 159 & 113 & 27 & 140 & $51.5 \%$ \\
\hline
\end{tabular}

Table 6. Experimental results for all cells undergoing cell grouping algorithm.

- $\quad$ Extracted cells: (2)

Number of extracted cells for categorizing cell groups.

- Unextracted cells: (3)

Number of unextracted cells.

- Cell groups: (4)

Number of categorized cell groups (which become the typical character cell)

- $\quad$ Cell types: (3)+(4)

Total cell types for both typical character cells and unextracted cells.

- $\quad$ Rate of reduction: $\{(3)+(4)\} /(1)$

Rate of reduction in cell types produced by the proposed method.

In the results for Data $\mathrm{A}$, the reduction rate was $39.4 \%$. In contrast, the results for Data $\mathrm{B}$ indicated a $51.5 \%$ reduction in cell types. From the results of the verification experiments, clearly the proposed method can reduce the number of cell types in OPCed layouts based on actual functional LSI. Accordingly, we were able to successfully achieve an improvement in extraction efficiency of approximately $60 \%$. Thus, for actual functional LSIs, the proposed method can also greatly reduce the variety in deformed patterns with OPC features.

\section{Conclusion}

In this research, we have proposed an approach to improving pattern extraction efficiency for character projection lithography on random logic devices by employing OPC optimization. Our proposed method is able to realize reductions in the variety of patterns 
deformed by the complicated OPC through two developed algorithms: (1) a cell grouping algorithm that categorizes differentiated cells and extracts some typical cell groups, and (2) an OPC optimization algorithm that regards the cells in a group as one typical cell and corrects for the OPC features of a typical cell to form a CP aperture mask. As the results of conducted experiments demonstrate with 40 types test patterns, the proposed method realized a 30\% improvement in extraction efficiency. Moreover, in conducted verification experiments with two full-chip level OPCed layouts based on actual functional LSI, we successfully achieved an improvement in extraction efficiency of approximately $60 \%$. In addition, in order to put these research results to practical use for CPL, it is necessary to conduct verifications that compare EB shots.

In the future, as the trend for semiconductor devices to become increasingly smaller in size is likely to continue, it is expected that mask costs and TATs will continue to rise. Technologies that reduce mask costs and TATs are extremely important key factors for the manufacture of system LSIs. We believe that our proposed method may show a new way of overcoming mask problems.

\section{Acknowledgments}

This work was supported by the Association of Super-Advanced Electronics Technologies (ASET) and the New Energy and Industrial Technology Development Organization (NEDO). The authors would like to thank the Semiconductor Technology Academic Research Center (STARC) for their support with the pattern data.

\section{References}

Nakayama, Y.; Okazaki, S.; Saitou, N. \& Wakabayashi, H. (1990). Electron-beam cell projection lithography: A new high-throughput electron-beam direct-writing technology using a specially tailored Si aperture, Journal of Vacuum Science $\mathcal{E}$ Technology B: Microelectronics and Nanometer Structures, Vol. 8, No. 6, pp. 1836-1840, ISSN: 10711023.

Pfeiffer, H., C. (1978). Variable spot shaping for electron beam lithography, Journal of Vacuum Science \& Technology B: Microelectronics and Nanometer Structures, Vol. 15, No. 3, pp. 887-890, ISSN: 10711023.

Matsunawa, T.; Nosato, H.; Sakanashi, H.; Murakawa, M.; Murata, N.; Terasawa, T.; Tanaka, T.; Yoshioka, N.; Suga, O. \& Higuchi, T. (2005). The novel approach for optical proximity correction using genetic algorithms, Proceedings of SPIE, Vol. 5992, pp. 541-549, SPIE, ISBN: 9780819460141.

Matsunawa, T.; Nosato, H.; Sakanashi, H.; Murakawa, Takahashi, E.; Terasawa, T.; Tanaka, T.; Suga, O. \& Higuchi, T. (2007). Adaptive optical proximity correction using an optimization method, Proceedings of IEEE 7th International Conference on Computer and Information Technology (CIT 2007), pp. 853-858, IEEE, ISBN: 0769529836.

ASET. (2006). Frontier Research on Optimization of Design, Drawing and Inspection for 45nmhp System LSI, FY 2005 Annual Report, NEDO Japan, (in Japanese).

Nosato, H.; Matsunawa, T; Sakanashi, H; Murakawa, M. \& Higuchi, T. (2007). A Fast Method of Adaptive Optical Proximity Correction Using Region Segmentation, IPSJ Transactions on Mathematical Modeling and Its Applications, Vol. 48, pp. 75-85, IPSJ, ISSN: 03875806, (in Japanese).

Cantú-Paz, E. (1995). A Summary of Research on Parallel Genetic Algorithms, IlliGAL Report, No. 95007, Illinois Genetic Algorithms Lab., University of Illinois. 


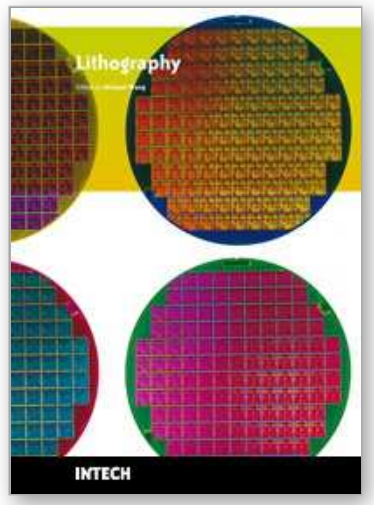

\author{
Lithography \\ Edited by Michael Wang
}

ISBN 978-953-307-064-3

Hard cover, 656 pages

Publisher InTech

Published online 01, February, 2010

Published in print edition February, 2010

Lithography, the fundamental fabrication process of semiconductor devices, plays a critical role in micro- and nano-fabrications and the revolution in high density integrated circuits. This book is the result of inspirations and contributions from many researchers worldwide. Although the inclusion of the book chapters may not be a complete representation of all lithographic arts, it does represent a good collection of contributions in this field. We hope readers will enjoy reading the book as much as we have enjoyed bringing it together. We would like to thank all contributors and authors of this book.

\title{
How to reference
}

In order to correctly reference this scholarly work, feel free to copy and paste the following:

Hirokazu Nosato, Hidenori Sakanashi, Masahiro Murakawa and Tetsuya Higuchi (2010). Improving the Efficiency of Pattern Extraction for Character Projection Lithography using OPC Optimization, Lithography, Michael Wang (Ed.), ISBN: 978-953-307-064-3, InTech, Available from:

http://www.intechopen.com/books/lithography/improving-the-efficiency-of-pattern-extraction-for-characterprojection-lithography-using-opc-optimi

\section{INTECH}

open science | open minds

\section{InTech Europe}

University Campus STeP Ri Slavka Krautzeka 83/A 51000 Rijeka, Croatia

Phone: +385 (51) 770447

Fax: +385 (51) 686166 www.intechopen.com

\section{InTech China}

Unit 405, Office Block, Hotel Equatorial Shanghai No.65, Yan An Road (West), Shanghai, 200040, China 中国上海市延安西路65号上海国际贵都大饭店办公楼 405 单元 Phone: +86-21-62489820

Fax: +86-21-62489821 
(C) 2010 The Author(s). Licensee IntechOpen. This chapter is distributed under the terms of the Creative Commons Attribution-NonCommercialShareAlike-3.0 License, which permits use, distribution and reproduction for non-commercial purposes, provided the original is properly cited and derivative works building on this content are distributed under the same license. 